\title{
Saúde mental do idoso: uma questão de saúde pública
}

Olga Laura Sena Almeida ${ }^{1}$

$\mathrm{N}$ esta edição da Revista Medicina (Ribeirão Preto) são publicados dois artigos que abordam a saúde mental do idoso, com destaque para a importância da identificação precoce de fatores de risco para depressão nessa faixa etária e as diferenças nos índices de mortalidade por suicídio em idosos nas diferentes regiões do Brasil.

A depressão em idosos pode ser considerada um problema de saúde pública por sua elevada prevalência nessa faixa etária e seu impacto na qualidade de vida e funcionalidade do idoso. É uma condição clínica que está frequentemente associada a multimorbidades e ao aumento do risco de suicídio e mortalidade em geral nessa população ${ }^{1}$. Apesar da sua relevância, o grau de conhecimento dos profissionais de saúde envolvidos no cuidado ao idoso com relação à identificação de vulnerabilidades emocionais ou psicossociais ainda é limitado, refletindo a insuficiência de publicações científicas sobre o tema. Os idosos são muitas vezes excluídos de ensaios clínicos robustos e os resultados desses estudos são extrapolados para essa população com limitações. Deste modo, estudos que abordem as peculiaridades da depressão em idosos são de extrema importância para a melhoria da qualidade da assistência prestada a esses pacientes².

No artigo "Prevalence and factors associated with depression in the elderly: a cross-sectional study"3, os autores avaliaram a prevalência de depressão e os fatores associados a essa condição em idosos de uma capital brasileira (Cuiabá, Mato Grosso). Baixa escolaridade, dependência para as atividades de vida diária, presença de multimorbidades, alterações do sono, ocorrência de quedas e déficits visuais foram os fatores associados à depressão na população estudada. O estudo destacou que os fatores encontrados são prevalentes na população idosa e também potencialmente modificáveis, e que, uma vez identificados precocemente, podem ser adequadamente tratados, prevenindo a ocorrência de eventos depressivos que podem levar a prejuízos funcionais importantes, com perda de qualidade de vida e aumento do risco de morte nessa faixa etária. Medidas de socialização, estímulos à atividade física regular que melhorem o equilíbrio e reduzam o risco de sarcopenia e síndrome da fragilidade, e investimentos em educação, são algumas das intervenções destacadas pelos autores que podem ser realizadas como políticas públicas para mudança de hábitos e redução do risco de depressão em idosos.

A depressão em idosos pode se manifestar de maneira atípica, o que pode dificultar o diagnóstico e o tratamento adequados, aumentando os riscos de complicações. Além disso, idosos frequentemente apresentam multimorbidades, polifarmácia e déficit cognitivo, que aumentam o risco de eventos adversos mais graves ${ }^{4}$. Dentre as possíveis complicações relacionadas à depressão no idoso, o suicídio merece destaque, especialmente em países em desenvolvimento, com renda média ou baixa, onde ocorrem $78 \%$ dos eventos de suicídio no mundo ${ }^{5}$.

O suicídio no idoso também é considerado um problema de saúde pública complexo, que precisa ser amplamente discutido e estudado. Dentre as complexidades relacionadas ao tema, pode-se destacar o aumento da letalidade em idosos em comparação a pessoas mais jovens, a falta de dados a respeito

1. Divisão de Clínica Médica Geral e Geriatria, Departamento de Clínica Médica, Faculdade de Medicina de Ribeirão Preto, Universidade de São Paulo (USP), Ribeirão Preto (SP), Brasil.

$\triangle$ Endereço para correspondência: Departamento de Clínica Médica. Avenida dos Bandeirantes, 3900, 6 andar do HC, Monte Alegre. CEP: $14048-900$. Ribeirão Preto (SP), Brasil. olga.almeida@usp.br 
da presença de pensamentos de morte como indicadores de risco nessa faixa etária, a dificuldade de acesso que os idosos têm para buscar assistência médica adequada e tratamento especializado e a escassez de estudos sobre suicídio nessa população ${ }^{6}$

O suicídio é um fenômeno mundial, mas há diferenças entre regiões e países no que diz respeito à idade, sexo e condição socioeconômica do indivíduo e do respectivo país, além de diferenças com relação ao método utilizado para o suicídio e o acesso aos cuidados de saúde. Muitos desses aspectos não costumam ser contemplados em pesquisas e uma abordagem mais profunda destacando essas diferenças regionais pode contribuir para o desenvolvimento de medidas de prevenção do suicídio mais efetivas, permitindo a intervenção precoce em idosos com maior risco ${ }^{5}$.

No artigo "Suicídio em idosos: índice e taxa de mortalidade nas capitais brasileiras no período de 2001 a $2015^{\prime \prime}$, os autores avaliaram o índice e a taxa de mortalidade por suicídio em idosos nas capitais brasileiras, destacando as regiões com as maiores taxas de suicídio e com o maior crescimento dos índices nas últimas décadas. O suicídio foi mais prevalente em homens, na faixa etária entre 60 e 69 anos e na região sudeste do país. Na análise dos resultados, os autores destacaram que há diferenças geográficas, climáticas, econômicas e educacionais que podem contribuir para as diferenças encontradas entre as regiões do país. Ressaltaram, ainda, que a maior taxa de mortalidade entre homens idosos pode se dever ao prejuízo das funções sociais que ocorre com o envelhecimento e que são culturalmente atribuídas à hegemonia da masculinidade, além do uso de estratégias de suicídio mais efetivas.

A literatura mostra que o suicídio é mais comum em pessoas do sexo masculino e costuma estar relacionado a doenças psiquiátricas. Entre elas, a depressão e a psicose são os fatores de risco mais relevantes, além de ansiedade e transtornos de personalidade ${ }^{5}$. Porém, apesar dos aspectos psicológicos serem importantes, mais atenção deve ser dada às causas físicas subjacentes à tentativa de suicídio, especialmente em idosos. Há estudos que mostram que o diagnóstico de uma doença física é a causa mais comum de suicídio em pessoas idosas, sendo considerado um fator de risco para o suicídio nessa população .

Ainda há um número significativo de subnotificação de depressão e suicídio em idosos e a discussão ampla do assunto precisa ser realizada na comunidade científica para melhoria e desenvolvimento de medidas de prevenção mais efetivas nessa população $0^{5}$. Políticas públicas de suporte mental contínuo são necessárias para prevenir a depressão em pacientes idosos que apresentam multimorbidades, além de medidas que reduzam a carga de sintomas físicos e deficiências funcionais, reduzindo o risco do desenvolvimento de pensamentos e comportamentos suicidas. Deve-se investigar ativamente pensamentos suicidas e depressão em idosos com doenças físicas atendidos na atenção primária e, uma vez identificada a ideação suicida, o tratamento psicogeriátrico deve ser imediatamente instituído ${ }^{8}$. Além disso, a restrição do acesso a ferramentas utilizadas para o suicídio e o treinamento de médicos da atenção primária e demais profissionais da área da saúde para identificação de idosos em risco devem ser estimulados como estratégias de prevenção ${ }^{5}$. Há dados na literatura mostrando que a utilização de rastreamento de depressão em idosos pode reduzir as taxas de suicídio nessa população, e a identificação de fatores de risco parece ser importante 8 .

A depressão e o suicídio em idosos representam um grave problema social e de saúde pública, e investimentos em pesquisas sobre o tema devem ser realizados, com ampla divulgação dos resultados à comunidade para auxílio no desenvolvimento de políticas públicas mais efetivas no cuidado à saúde mental do idoso, melhorando sua qualidade de vida e preservando a sua funcionalidade.

\section{REFERÊNCIAS}

1. Valiengo LCL, Stella F, Forlenza OV. Mood disorders in the elderly: prevalence, functional impact, and management challenges. Neuropsychiatr Dis Treat 2016; 12: 2105-2114.

2. Agüera-Ortiz L, Claver-Martín MD, Franco-Fernández MD et al. Depression in the Elderly. Consensus Statement of the Spanish Psychogeriatric Association. Front Psychiatry 2020; 11: 380.

3. Leite TSM, Fett CA, Stoppiglia LF, Neves T, Figueiredo KRFV, Rodrigues RAS, et al. Prevalence and factors associated with depression in the elderly: a cross-sectional 
study. Medicina (Ribeirão Preto). 2020;53(3):205-214. doi: https://doi.org/10.11606/issn.2176-7262.v53i3p205-214

4. Kok RM, Reynolds 3rd CF. Management of Depression in Older Adults: A Review. JAMA 2017; 317: 2114-2122.

5. Bachmann S. Epidemiology of Suicide and the Psychiatric Perspective. Int J Environ Res Public Health 2018; 15(7): 1425.

6. Orden KAV, Conwell Y. Issues in Research on Aging and Suicide. Aging Ment Health 2016; 20(2): 240-51.
7. Silva JVS, Santos Júnior CJS, Oliveira KCPN. Suicídio em idosos: índice e taxa de mortalidade nas capitais brasileiras no período de 2001 a 2015. Medicina (Ribeirão Preto). 2020;53(3):215-222. doi: https://doi.org/10.11606/issn.2176-7262.v53i3p215-222

8. Kim SH, Kim HJ, Oh SH et al. Analysis of attempted suicide episodes presenting to the emergency department: comparison of young, middle aged and older people. Int J Ment Health Syst 2020; 14: 46. 